\title{
Characterizing longitudinal white matter development during early childhood
}

\author{
Douglas C. Dean III · Jonathan O'Muircheartaigh • \\ Holly Dirks • Nicole Waskiewicz $\cdot$ Lindsay Walker • \\ Ellen Doernberg • Irene Piryatinsky $\cdot$ Sean C. L. Deoni
}

Received: 30 January 2014/ Accepted: 19 March 2014/Published online: 8 April 2014

(c) The Author(s) 2014. This article is published with open access at Springerlink.com

\begin{abstract}
Post-mortem studies have shown the maturation of the brain's myelinated white matter, crucial for efficient and coordinated brain communication, follows a nonlinear spatio-temporal pattern that corresponds with the onset and refinement of cognitive functions and behaviors. Unfortunately, investigation of myelination in vivo is challenging and, thus, little is known about the normative pattern of myelination, or its association with functional development. Using a novel quantitative magnetic resonance imaging technique sensitive to myelin we examined longitudinal white matter development in 108 typically developing children ranging in age from 2.5 months to 5.5 years. Using nonlinear mixed effects modeling, we provide the first in vivo longitudinal description of myelin water fraction development. Moreover, we show distinct male and female developmental patterns, and demonstrate significant relationships between myelin content and measures of cognitive function. These findings advance a new understanding of healthy brain development and provide a foundation from which to assess atypical development.
\end{abstract}

Electronic supplementary material The online version of this article (doi:10.1007/s00429-014-0763-3) contains supplementary material, which is available to authorized users.

D. C. Dean III $(\bowtie) \cdot$ J. O’Muircheartaigh $\cdot$ H. Dirks .

N. Waskiewicz · L. Walker · E. Doernberg · I. Piryatinsky ·

S. C. L. Deoni

Advanced Baby Imaging Laboratory, School of Engineering,

Brown University, Providence, RI 02912, USA

e-mail: douglas_dean_iii@brown.edu

J. O'Muircheartaigh

Department of Neuroimaging, King's College London, Institute

of Psychiatry, De Crespigny Park, London SE5 8AF, UK
Keywords Brain development - White matter development $\cdot$ Magnetic resonance imaging $\cdot$ Myelin water fraction

\section{Introduction}

Human brain development is a multifaceted process that begins in utero and continues throughout childhood and into early adulthood. Despite this protracted timeline, postnatal neurodevelopment during the first 5 years of life is one of the most active stages of brain development (Pujol et al. 2006). During this period neural migration, synapse generation, dendritic sprouting, axonal pruning, and the elaboration of the myelin sheath (myelination) shape, organize, and provide the neural architecture necessary for behavioral and cognitive functioning (Durston and Casey 2006). While each of these processes is essential for healthy brain function, the establishment of the myelinated white matter is integral to establishing the efficient brain communication pathways that are essential for higher order function (Fields 2008). Although myelination is believed to mirror behavioral development (Pujol et al. 2006), with the myelination of sub-serving brain networks occurring alongside the onset and refinement of behavioral and cognitive functions, the relationships between myelination and functional development remain poorly understood. Furthermore, many behavioral and psychiatric disorders are now believed to emerge during early neurodevelopment (Hüppi 2008) and have been associated with aberrant white matter development and myelination (Fields 2008). Thus, improved understanding of typical myelin development during infancy and early childhood is central to provide valuable insight into the processes that underline both typical and atypical development. 
Advanced magnetic resonance imaging (MRI) techniques have greatly contributed to our understanding of the gross and microstructural changes associated with brain maturation (Giedd and Rapoport 2010). For example, the temporal changes in the gray/white matter contrast in conventional $T_{1}$ - and $T_{2}$-weighted MR images has been used to illustrate the dynamic patterns of neurodevelopment (Paus et al. 2001). Diffusion tensor (DT)-MRI parameters, including mean and radial diffusivity (MD and $\mathrm{RD}$, respectively), and fractional anisotropy (FA), which describe local measures of water diffusion and anisotropy, have been shown to change throughout childhood and adolescence (Lebel et al. 2008). Magnetization transfer (MT) imaging, believed to be sensitive to myelin content, has additionally been used to examine the in vivo changes of white matter myelination (Rademacher et al. 1999). While alterations of these MR parameters are often attributed to the emergence and maturation of myelin, they do not directly measure myelin (Paus et al. 2001) and are influenced by a broad range of other microstructural changes (Jones et al. 2013).

Beyond the technological difficulty in non-invasively imaging myelination, few imaging studies have examined longitudinal neurodevelopment across the first years of life. Rather, prior studies have either been cross-sectional in nature (Giedd et al. 1996; Casey et al. 2005) and/or have focused on only the first 1-2 years of life (Gilmore et al. 2012; Sadeghi et al. 2013), or after the age of 4 (Giedd et al. 1999; Lenroot et al. 2007; Lebel and Beaulieu 2011). Although cross-sectional studies are informative, they are inherently insensitive to individual differences, an important aspect in understanding the heterogeneity associated with both normative development, as well as in developmental disorders (Casey et al. 2005). Longitudinal study designs, on the other hand, afford a more thorough characterization of brain development. Through the acquisition of multiple measurements from the same individual, longitudinal analyses allow individual differences to be examined (Casey et al. 2005) and are, therefore, critical to understanding the developmental trajectory of brain development. However, as current longitudinal MRI studies have been limited to infancy (Gilmore et al. 2012; Sadeghi et al. 2013), or older childhood and adolescence (Giedd et al. 1999; Lenroot et al. 2007; Lebel and Beaulieu 2011), these studies fail to encompass the most rapid and dynamic period of neurodevelopment (Pujol et al. 2006), which also coincides with the emergence of many developmental neuropsychiatric disorders, including autism spectrum and attention deficit/hyperactivity disorder.

Here, we aimed to perform the first longitudinal examination of normative brain development in healthy, typically developing children between 2.5 months and 5.5 years. More specifically, we examined the rapid, nonlinear progression of myelination using a quantitative multicomponent relaxometry (MCR) technique that is sensitive to the water trapped between the lipid bilayers of the myelin sheath (MacKay et al. 1994; Deoni et al. 2008). Repeated MRI scans were acquired using the rapid mcDESPOT (multicomponent driven equilibrium single pulse observation of $T_{1}$ and $T_{2}$ ) (Deoni et al. 2008) MCR technique in a large cohort of 108 typically developing children. Longitudinal developmental trajectories from 28 brain regions and major white matter pathways are presented. We utilize a nonlinear mixed effects modeling framework and a fourparameter Gompertz function (Dean et al. 2014a) to explicitly model the nonlinear growth patterns of the myelin water fraction $\left(\mathrm{VF}_{\mathrm{M}}\right)$, a surrogate measure of myelin content, for each individual (random effects) and the overall population (fixed effects). Parameter estimates from this nonlinear fitting lead to the characterization of a normative model of $\mathrm{VF}_{\mathrm{M}}$ development, which can then be used to reconstruct typical $\mathrm{VF}_{\mathrm{M}}$ growth and growth rate patterns. Finally, modeled trajectories, coupled with longitudinal scores of cognition and behavior, were examined to identify relationships between $\mathrm{VF}_{\mathrm{M}}$ and functional development. Quantitative analysis of these data provides further insight into the processes of brain maturation and its relationship to cognitive evolution, and provides a foundation for future studies of atypical development.

\section{Methods}

Subjects

Subjects for this study consisted of 108 healthy, typically developing children recruited to take part in an ongoing longitudinal study investigating the relationships between myelin maturation and behavioral development (Deoni et al. 2012). Parental consent was obtained in accordance to ethics approval from the host institution's Institutional Review Board. Inclusion criteria consisted of uncomplicated (i.e., no preeclampsia, and APGAR scores $>8$ ) singleton birth between 37 and 42 weeks; no abnormalities on fetal ultrasound; no exposure to alcohol or illicit drugs during pregnancy; no familial history of major psychiatric or depressive illnesses; no diagnosis of major psychiatric, depressive or learning disorder in the participant; and no pre-existing neurological conditions or major head trauma.

Subjects were composed of 51 females and 57 males that were between 70 and 1,928 days (GC, gestationally corrected to a 40 week gestation) of age at recruitment. An age-dependent scanning schedule was used to determine when children would return for follow-up MRI sessions. Children under 2 years of age returned every 6 months, while children over 2 years of age returned yearly (Dean 
et al. 2014b). Additional demographic information is provided in Supplementary Table 1. No statistical differences between birth weight, birth height, and gestational period were found between the age groups.

Image acquisition

Scanning of children under 4 years of age was performed during natural (i.e., non-sedated) sleep (Dean et al. 2014b). Children over this age were scanned while watching a favorite movie or TV show. Imaging slew rates and gradient amplitudes were reduced to approximately 30 and $75 \%$ of their maximal values to reduce the acoustic noise levels of the MRI scanner and ensure sleeping children remained asleep for the duration of the scan. Additional passive measures, including electrodynamic headphones (MR Confon, Germany) and a removable sound-insulating foam insert (Ultra Barrier HD Composite, UltraBarrier USA) that conformed to the inside of the scanner bore, provided further acoustic noise attenuation. To reduce subtle body movement, children were swaddled with appropriately sized MedVac vacuum immobilization bags (CFI Medical Solutions, USA).

All image data were acquired on the same 3T Siemens Tim Trio MRI scanner with a 12-channel head RF coil array. Optimized pediatric mcDESPOT protocols previously developed for varying head size and age range were used (Deoni et al. 2012). In general, the mcDEPSOT imaging protocol consists of $8 T_{1}$-weighted spoiled gradient echo (SPGR or spoiled FLASH) images, 2 inversion-prepared (IR)-SPGR images, and $16 T_{1} / T_{2}$-weighted balanced steadysteady state free precession (bSSFP or TrueFISP) images. SPGR and bSSFP images were acquired at varying flip angles, while the bSSFP images were collected at two-phase increments $\left(0^{\circ}\right.$ and $\left.180^{\circ}\right)$. Field of view and the image matrix size were adjusted so that whole-brain coverage was achieved while maintaining a consistent $1.8 \times 1.8 \times 1.8$ $\mathrm{mm}^{3}$ voxel resolution. Total acquisition time for all protocols was $<30 \mathrm{~min}$. Additional information about the imaging parameters used is included as supplementary information (Supplementary Table 2).

In addition to the mcDESPOT data, a higher resolution $\left(1.5 \times 1.5 \times 1.5 \mathrm{~mm}^{3}\right) T_{1}$-weighted image and resting state functional MRI data were acquired if the child remained asleep and cooperative.

$\mathrm{VF}_{\mathrm{M}}$ measurements

Following successful MR data acquisition, the 26 individual SPGR, IR-SPGR, and bSSFP images for each participant were linearly co-registered to account for subtle head movement and non-parenchyma voxels removed using a deformable model approach (Smith et al. 2004). $\mathrm{VF}_{\mathrm{M}}$ values were then calculated at each image voxel by fitting the SPGR and bSSFP data to a 3-pool tissue model that estimates the volume fractions and relaxation times for intra/extra-axonal water, myelin-associated water, and non-exchanging free water (Deoni et al. 2013). Quantification of the properties from these microstructural water compartments distinguishes each component and therefore provides unique information about the underlying microstructure. Additional corrections for radio-frequency flip angle $\left(B_{1}\right)$ and main magnetic field $\left(B_{0}\right)$ inhomogeneities were also performed (Deoni 2010).

\section{Registration of longitudinal measurements}

Independently registering longitudinal measurements to a common template may introduce subtle inconsistencies when not accounting for the repeated regularity of subjectspecific measurements (Aubert-Broche et al. 2013). To minimize this possible source of variability, a longitudinal registration pipeline was developed to align each individual's measurements into a common analysis space. This registration was performed using the high flip angle SPGR image with the resulting transformations subsequently applied to the quantitative $\mathrm{VF}_{\mathrm{M}}$ maps.

For each subject, a $T_{1}$-weighted template was created from the acquired longitudinal time points using symmetric diffeomorphic normalization and a cross-correlation similarity metric (Avants et al. 2008). An initial rigid registration between the subject's high flip angle SPGR images and a study-specific template previously created from $T_{1^{-}}$ weighted data of children of the same age range and approximately in the space of the MNI template (Deoni et al. 2012) was performed to bring the images into a rough alignment. Next, a subject-specific template was created from these roughly aligned SPGR images using the ANTs buildtemplateparallel.sh script that is included in the ANTs package. The subject-specific template was then nonlinearly registered to the study template using symmetric diffeomorphic normalization. Finally, individual parameter maps for each time point were transformed into the overall study template by concatenating the transformations from native space to the subject-specific template space and from subject-specific template space to the overall study template in a single interpolation step (Supplementary Fig. 1).

Following successful registration of each individual dataset, $\mathrm{VF}_{\mathrm{M}}$ images were smoothed with a conservative 3-mm (full width at half maximum) Gaussian kernel to accommodate subtle structural variations among the individual subjects that were not handled by the registration procedure.

Longitudinal developmental trajectories

While there exists high individual developmental variation, distinct patterns of structural development can be observed 
and provide insight to brain maturation. For this reason we constructed longitudinal developmental trajectories of $\mathrm{VF}_{\mathrm{M}}$ from our cohort of subjects. Anatomically co-registered masks, obtained from the MNI database (Mazziotta et al. 2001) and the Johns Hopkins University DTI-based white matter atlas (Mori et al. 2009), were applied to the individual $\mathrm{VF}_{\mathrm{M}}$ data. In total, 28 regions were examined (detailed in Supplementary Fig. 2). For each longitudinal time point and anatomical region, mean $\mathrm{VF}_{\mathrm{M}}$ values were extracted and plotted against the subject's gestation-corrected age.

Modeling longitudinal $\mathrm{VF}_{\mathrm{M}}$ development

Nonlinear mixed effects modeling was used to characterize $\mathrm{VF}_{\mathrm{M}}$ development due to its ability to account for repeated measurements from the same individual, and non-equally distributed data (Lindstrom and Bates 1990). Modified Gompertz growth curves of the form:

$\mathrm{VF}_{\mathrm{M}}=\alpha \exp \left(-\exp \left(\beta-\gamma_{\mathrm{t}}\right)+\delta_{\mathrm{t}}\right)$

were fit to the developmental trajectories from the 28 regions (MATLAB 2013a, Natick, MA). This sigmoidal model was chosen to describe the nonlinear growth patterns of early neurodevelopment as this model has previously been shown to best represent $\mathrm{VF}_{\mathrm{M}}$ development in a cross-sectional cohort and offers informative parameterization of the developmental trajectory (Dean et al. 2014a, b); where $\alpha$ controls for the overall size of the curve, $\beta$ controls for the initial lag of the trajectory, and the parameters $\gamma$ and $\delta$ both correspond to growth rates. Supplementary Fig. 3 illustrates the effect that each parameter has on an example model growth curve.

Within this model formulation, the set of parameters, $(\alpha, \beta, \gamma, \delta)$, corresponds to the sum of the fixed and random effects. The set of parameters corresponding to the fixed effects characterize the overall population trajectory, while parameters that describe the random effects are estimated for each individual and allow the estimation of subject-specific growth curves (Lindstrom and Bates 1990).

In addition to modeling population (fixed effects) and individual (random effects) growth, the growth rate of $\mathrm{VF}_{\mathrm{M}}$ during this time period can also be examined. Using the Gompertz growth parameter estimates from above, we compute the rate of change of $\mathrm{VF}_{\mathrm{M}}$ by taking the time derivative of Eq. (1),

$\partial_{\mathrm{t}} \mathrm{VF}_{\mathrm{M}}=\alpha \exp \left(-\exp \left(\beta-\gamma_{\mathrm{t}}\right)\right)\left[\gamma \exp \left(\beta-\gamma_{\mathrm{t}}\right)+\delta\right]$

From this equation and using the estimates of the fixed and random effect growth parameters, both population and individual growth rates curves can be calculated.
Sex-related $\mathrm{VF}_{\mathrm{M}}$ developmental differences

As gender-related developmental differences are often reported to occur during early neurodevelopment (Lenroot et al. 2007), we sought to examine if gender differences in $\mathrm{VF}_{\mathrm{M}}$ were apparent. Nonlinear mixed model fits of the regional growth trajectories to the modified Gompertz equation (Eq. 1) were independently performed on genderseparated longitudinal data. $F$-statistics were used to compare the residuals of the nonlinear fitting of sex-separated and sex-combined developmental trajectories, while two-sided $t$-statistics were used to compare male and female modified Gompertz parameter estimates. Significance was defined to be $p<0.05$ corrected for the familywise error rate using Holm-Bonferroni method (Holm 1979).

Investigation of $\mathrm{VF}_{\mathrm{M}}$ development and cognition

To elucidate the association between myelination and cognitive development, we performed linear regressions between longitudinal $\mathrm{VF}_{\mathrm{M}}$ data and repeated measures of age-appropriate assessments of motor control, language, and visual reception. Within 1 week of successful acquisition of MRI data, children were administered the Mullen Scales of Early Learning (Mullen 1995) by a trained researcher. This developmental assessment battery provided five scales of cognitive and behavioral ability (gross motor, fine motor, expressive language, receptive language, and visual reception) from which we could interrogate the inherent relationship between structural and functional development. Due to the appropriate age range for the exam and a known ceiling effect inherent to the Mullen battery (Mullen 1995; O'Muircheartaigh et al. 2013), we restricted our analysis to a subset of 69 datasets in which the final MRI scan was acquired from children under $1080 \mathrm{GC}$ days (or $N$ months). (Supplementary Table S3).

For each individual's raw Mullen's scores, an index of cognitive change was computed using the following formula,

$\Delta_{\mathrm{M}}=\frac{M_{\mathrm{f}}-M_{\mathrm{i}}}{M_{\mathrm{f}}+M_{\mathrm{i}}}$,

where $M_{\mathrm{f}}$ and $M_{\mathrm{i}}$ represent the raw Mullen's scores measured at the subject's final and initial cognitive assessment, respectively, and $\Delta_{M}$ represents the normalized measure of cognitive change between these two time points. Similarly, for each region of interest, subject-specific indices of $\mathrm{VF}_{\mathrm{M}}$ change were computed, 
$\Delta_{\mathrm{VF}_{\mathrm{M}}}=\frac{\mathrm{VF}_{\mathrm{M}}^{\mathrm{f}}-\mathrm{VF}_{\mathrm{M}}^{\mathrm{i}}}{\mathrm{VF}_{\mathrm{M}}^{\mathrm{f}}+\mathrm{VF}_{\mathrm{M}_{\mathrm{i}}}^{\mathrm{i}}}$,

where $V F_{M}^{f}$ and $V F_{M}^{i}$ represent final and initial $V F_{M}$ values calculated from the subject-specific $\mathrm{VF}_{\mathrm{M}}$ Gompertz growth curves (Eq. 1) at the subject's final and initial age, respectively, and $\Delta_{\mathrm{VF}_{\mathrm{M}}}$ represents the normalized change in modeled $\mathrm{VF}_{\mathrm{M}}$ between the final and initial time points. Relationships between $\Delta_{\mathrm{VF}_{\mathrm{M}}}$ and each $\Delta_{\mathrm{M}}$ (gross and fine motor, expressive and receptive language, and visual reception) as well as their interactions with mean age, were tested using a multivariate general linear model (GLM), correcting for the difference between final and initial ages, and the mean age of the time points. The inclusion of these two covariates within this GLM was important as both raw cognitive measures and $\mathrm{VF}_{\mathrm{M}}$ are mediated by age
(O'Muircheartaigh et al. 2013). In addition, combining all the cognitive scales into a single GLM allowed us to partially account for the correlation between each of the cognitive measures (Mullen 1995). This model was tested non-parametrically using permutation testing (FSL's randomize) and significance was defined to be $p<0.05$, corrected for the familywise error rate.

\section{Results}

A total of 260 mcDESPOT MRI datasets were acquired from the 108 participants: 73 subjects were scanned twice, 26 were scanned three times, and 9 were scanned 4 times. Matched coronal, sagittal, and coronal slices through the mean $\mathrm{VF}_{\mathrm{M}}$ maps for representative age groups are shown in
Fig. 1 Top row: ages of the 108 subjects at each scan. Each row denotes an individual subject and the repeated measurements are connected with a dashed line. Males (blue) and females (green) are additionally distinguished. Bottom row: mean mcDESPOT $\mathrm{VF}_{\mathrm{M}}$ maps at $3,6,9,12,24,36,48$, and 60 months of age illustrating the development of myelinated white matter throughout the brain
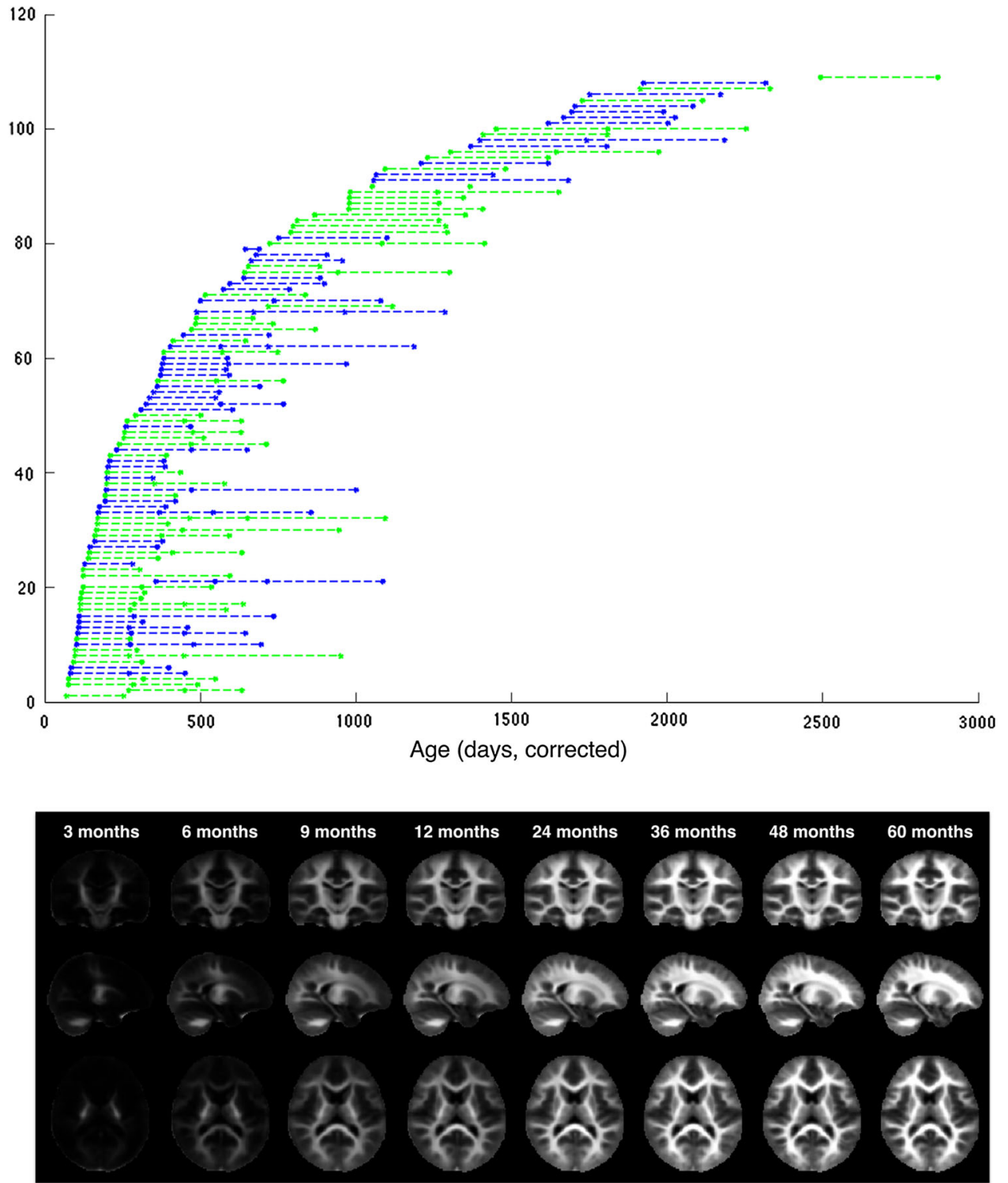

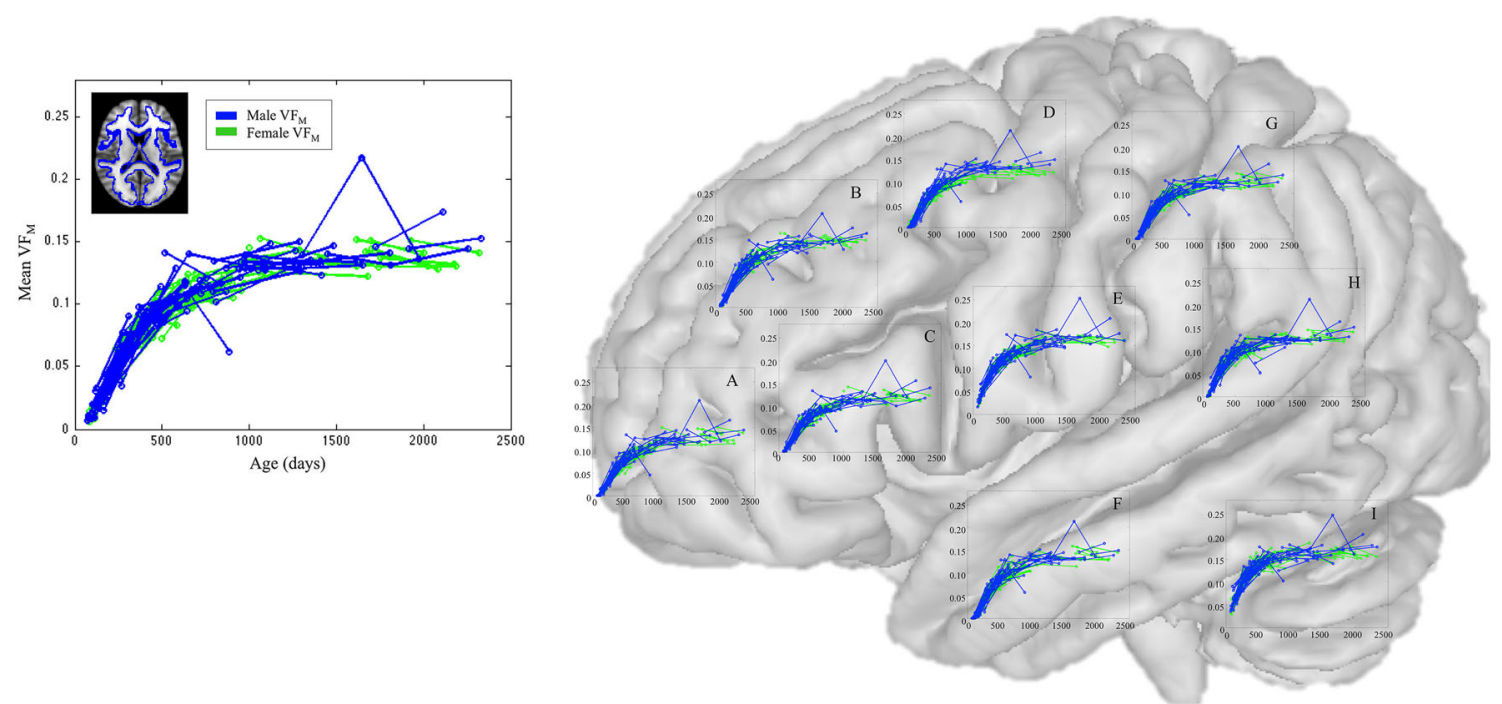

Fig. 2 Representative mean $\mathrm{VF}_{\mathrm{M}}$ developmental trajectories. Repeated measurements for each subject are connected with a straight line. Developmental trajectories are observed to follow a "S" shape pattern that is characteristic to a sigmoidal function.

Fig. 1 and illustrate the development of myelinated white matter throughout the brain.

Longitudinal $\mathrm{VF}_{\mathrm{M}}$ developmental trajectories for each of the 28 investigated regions (Supplementary Fig. 2) are shown in Fig. 2. Subject-specific measurements are connected with a straight line between each repeated time point. $\mathrm{VF}_{\mathrm{M}}$ increases nonlinearly with age, following a characteristic sigmoidal pattern with more rapid changes at early ages and slower development at older ages. This temporal pattern, beginning in deep and progressing to superficial white matter in a caudal-rostral direction, qualitatively agrees with previously described histological trajectories (Flechsig 1901; Yakovlev and Lecours 1967). It is noteworthy that although regional developmental trajectories were similar in profile, distinct temporal patterns are observed for specific regions. For example, frontal white matter is observed to have a longer lag in development compared to occipital and parietal white matter regions, which is in agreement with the frontal lobes being a late developing brain region.

Nonlinear mixed modeling of developmental trajectories

The mixed effect modeling framework was applied to the 28 regional $\mathrm{VF}_{\mathrm{M}}$ trajectories to obtain Gompertz growth curve parameter estimates of both population fixed and subject-specific random effects. Representative modeled developmental trajectories are shown in Fig. 3, with overall population curves overlaid on top of subject-specific developmental trajectories. Additional trajectories are
Anatomical locations associated with each graph: $A$ frontal lobe white matter, $B$ caudate, $C$ insula, $D$ putamen, $E$ thalamus, $F$ temporal lobe white matter, $G$ parietal lobe white matter, $H$ occipital lobe white matter, $I$ cerebellar white matter

provided in Supplementary Figure 4. These plots illustrate both the degree of individual variability of $\mathrm{VF}_{\mathrm{M}}$ development, as well as the ability of the modeling framework to capture the population's overall growth pattern. Moreover, mixed effects modeling provides standard error estimates of the fixed parameters, allowing derivation of confidence intervals of the developmental trajectory (Fig. 4).

Using the described population Gompertz parameter estimates, curves of the $\mathrm{VF}_{\mathrm{M}}$ growth rate were also derived; indicating how fast $\mathrm{VF}_{\mathrm{M}}$ changes over time. The rate of myelination is most intense at earlier ages for core white matter, whereas the fastest rate of myelin development occurs later in peripheral cortical regions (Fig. 4).

\section{Gender differences}

Results from comparing the nonlinear mixed effect models fitting of gender-separated data and the male/female pairwise $t$ tests between estimated Gompertz parameters are shown in Supplementary Table 4. F-statistics revealed no differences in the developmental trajectory residuals between the gender-separated and combined data. However, pairwise differences between male and female fixedeffect Gompertz parameter estimates were observed to be significant (Supplementary Table 4). For example, males were found to have significantly larger $\alpha$ values in the splenium of the corpus callosum, corresponding to an increased maximal $\mathrm{VF}_{\mathrm{M}}$ value; while differences in other model parameters (development lag, $\beta$; initial development 

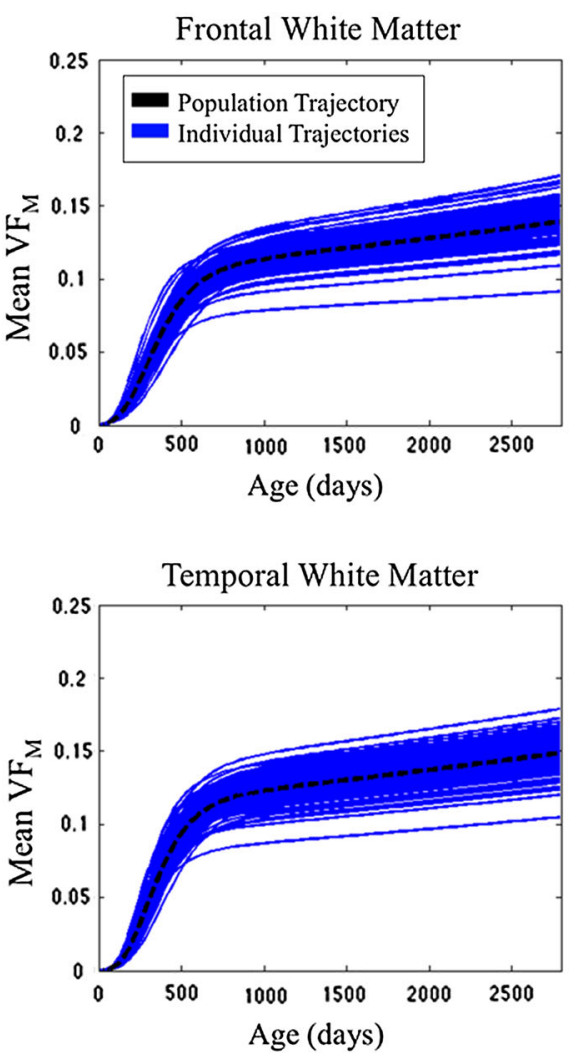

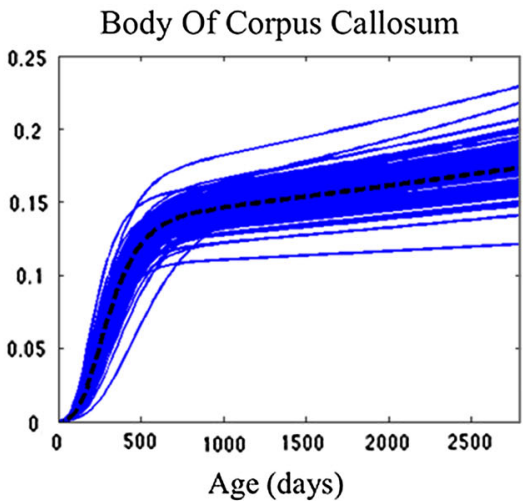

Parietal White Matter
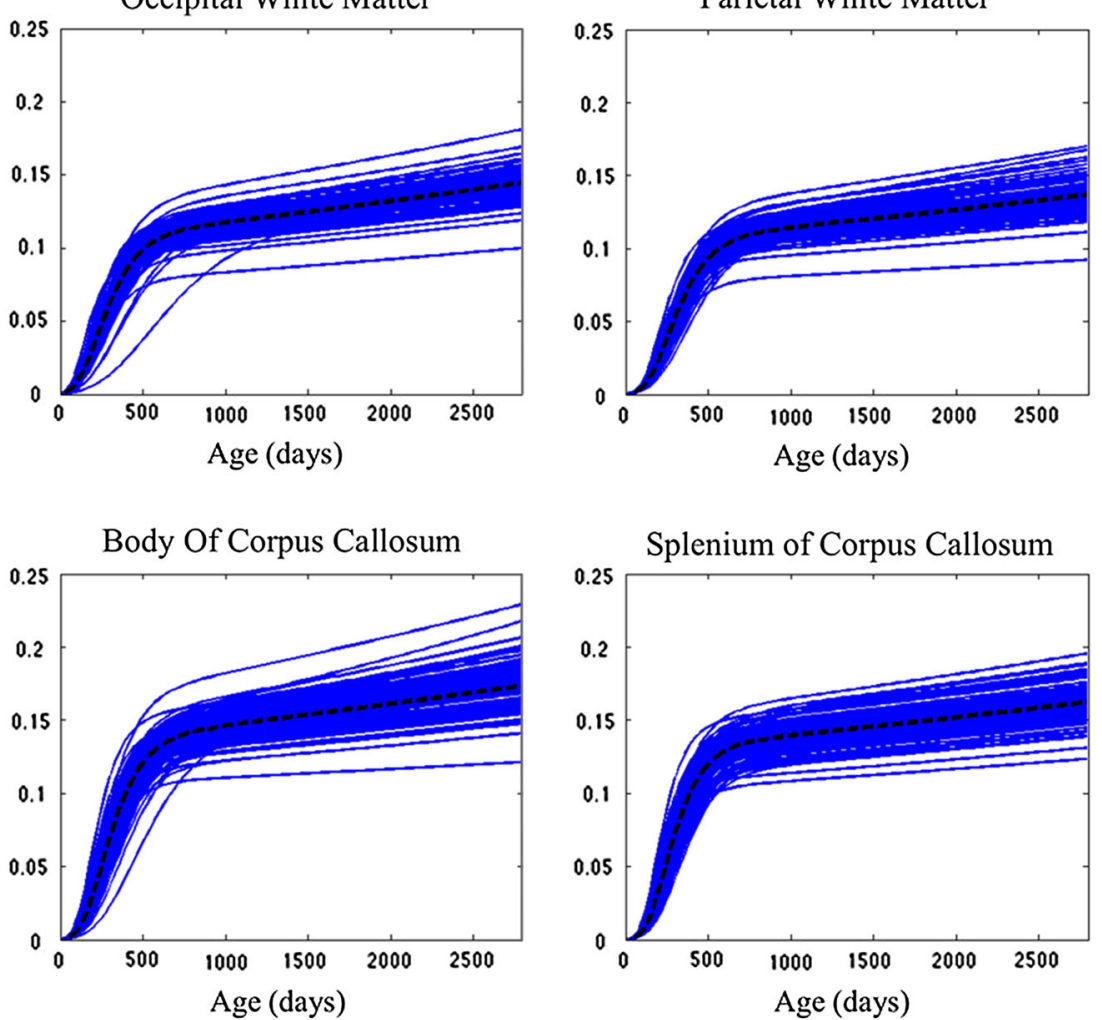

Fig. 3 Reconstructed modified Gompertz trajectories for each subject (blue curves) and the overall population (black dashed curve) for a representative subset of the investigated regions. Parameters for these

rate, $\gamma$; and secondary development rate, $\delta$ ) were found to vary between males and females in a majority of the regions investigated. On average, males were found to have a significantly larger secondary developmental rate $(\delta)$ than females, while females were found to have a larger increased maximal $\mathrm{VF}_{\mathrm{M}}(\alpha)$ than males. Differences between these parameters are reflected in the male and female developmental trajectories, as shown in Fig. 5 for the splenium of the corpus callosum.

\section{Developmental relationships with cognitive scores}

Longitudinal changes $\mathrm{VF}_{\mathrm{M}}$ were found to significantly ( $p<0.05$, corrected for multiple comparisons) correlate with changes in Mullen assessment scores. Representative plots illustrating the positive relationship between the change in $\mathrm{VF}_{\mathrm{M}}$ and the change in Mullen assessment scores are shown in the top row of Fig. 6 for the thalamus. Gross motor scores were correlated with $\mathrm{VF}_{\mathrm{M}}$ in areas of the basal ganglia, thalamus, cerebellum, and other core white matter tracts, while measures of visual reception were correlated with $\mathrm{VF}_{\mathrm{M}}$ in the posterior limb of the internal capsule, superior corona radiata, and the superior models were estimated by fitting the $\mathrm{VF}_{\mathrm{M}}$ profile to the modified Gompertz function as a function of gestationally corrected age using nonlinear mixed effects modeling

longitudinal fasciculus. Changes in receptive language scores were also correlated with changes in $\mathrm{VF}_{\mathrm{M}}$ in the cerebellum, thalamus, occipital white matter, and posterior thalamic radiations.

While no correlations were found to be significant between changes in expressive language or fine motor, the relationship between $\Delta_{\mathrm{VF}_{\mathrm{M}}}$ and $\Delta_{\mathrm{M}}$ for these scores was found to change over time. This age interaction is illustrated using a moving bin correlation in the bottom row of Fig. 6 for the thalamus. Prior to 350 days and following approximately 500 days, the correlation between expressive language $\Delta_{\mathrm{M}}$ and $\Delta_{\mathrm{VF}_{\mathrm{M}}}$ thalamus became increasingly negative, while in between this age range, the relationship appeared to become constant. The correlation between fine motor $\Delta_{\mathrm{M}}$ and $\Delta_{\mathrm{VF}_{\mathrm{M}}}$ thalamus became increasingly negative until approximately 500 days, before becoming increasingly positive. Similar age interactions with expressive language were observed in the posterior limb of the internal capsule and cerebellar white matter, while fine motor scores were detected to change over time in left hemispheric posterior limb of the internal capsule. A complete summary of the results from the general linear model analysis is provided in Supplementary Table 5. 
Frontal White Matter
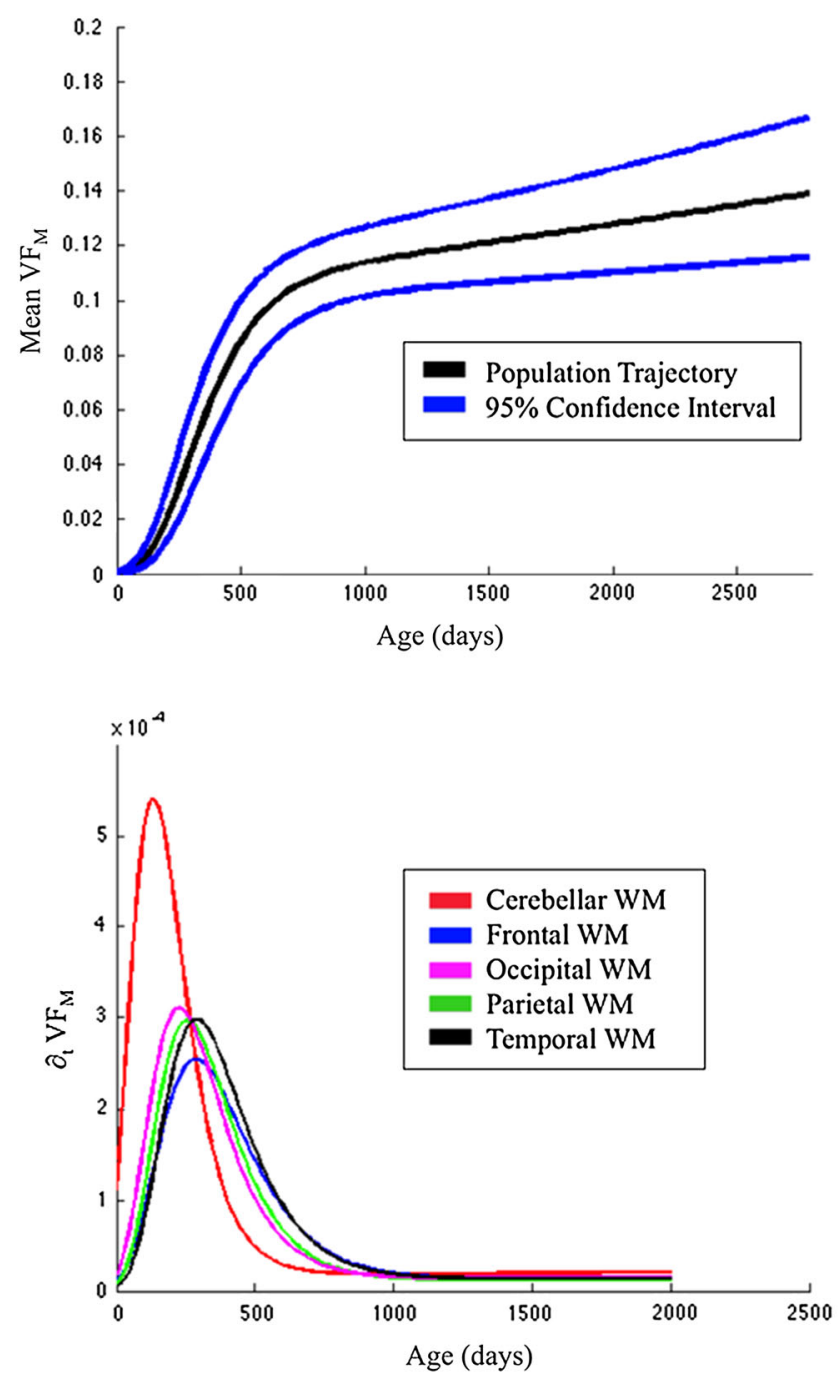

Fig. 4 Top Row: $95 \%$ confidence intervals for the frontal white matter and body of the corpus callosum. Using the standard error estimates from the nonlinear mixed effects modeling, confidence intervals of the population trajectory can be estimated. Bottom row: representative $\mathrm{VF}_{\mathrm{M}}$ growth rate curves. These curves were

\section{Discussion}

In this work, we have investigated longitudinal brain development from infancy through to early childhood using quantitative multicomponent relaxometry for the first time. From a large cohort of 108 typically developing male and female children, we have shown that regional $\mathrm{VF}_{\mathrm{M}}$ trajectories follow a nonlinear growth pattern, consistent with the myelination patterns of both histological studies (Flechsig 1901; Yakovlev and Lecours 1967) and our own prior cross-sectional $\mathrm{VF}_{\mathrm{M}}$ imaging studies of white matter development (Deoni et al. 2012). Using nonlinear mixed effects modeling, we have derived population and subject-specific growth model parameters that

\section{Body Of Corpus Callosum}
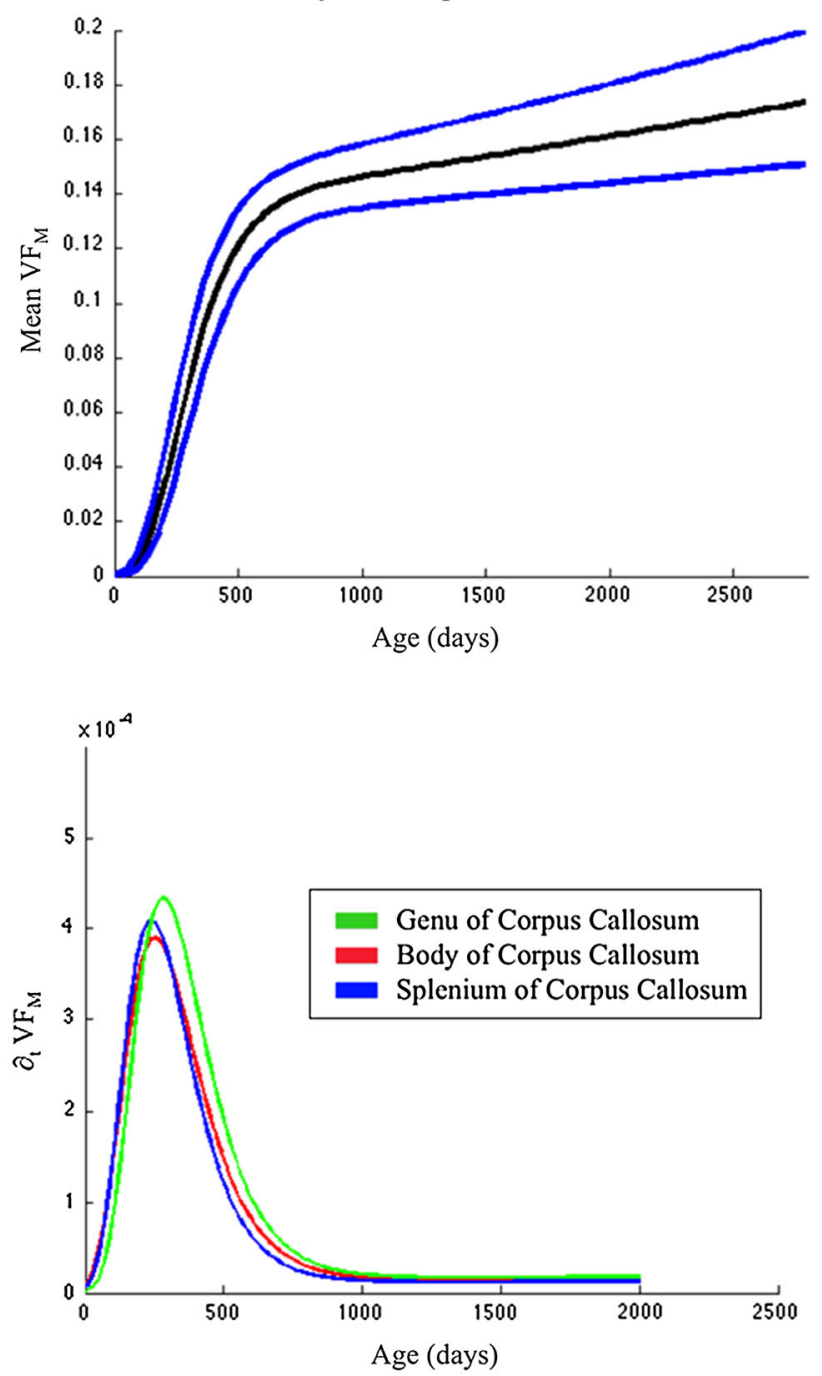

reconstructed by taking the time derivative of the modified Gompertz function (Eq. 2) and using the overall population estimates of the modified Gompertz parameters. Such curves are informative of the rate of change of the $\mathrm{VF}_{\mathrm{M}}$ with respect to age (time) and highlight the posterior-anterior developmental gradient of $\mathrm{VF}_{\mathrm{M}}$

characterize underlying $\mathrm{VF}_{\mathrm{M}}$ development and may be used to reconstruct normative reference growth and growth rate curves (as illustrated in Figs. 3, 4, 5). We have further examined the relationships between $\mathrm{VF}_{\mathrm{M}}$ maturation and cognitive development. Our results show a strong correlation between changes in $\mathrm{VF}_{\mathrm{M}}$ and scales of visual reception, gross motor, and receptive language, while the relationships between $\mathrm{VF}_{\mathrm{M}}$ and expressive language and fine motor were found to change in time. These findings support previous reports of the concomitant emergence of myelinated white matter and cognitive function and more importantly, may reveal a window into the timing of biological changes that shape behavioral development. 


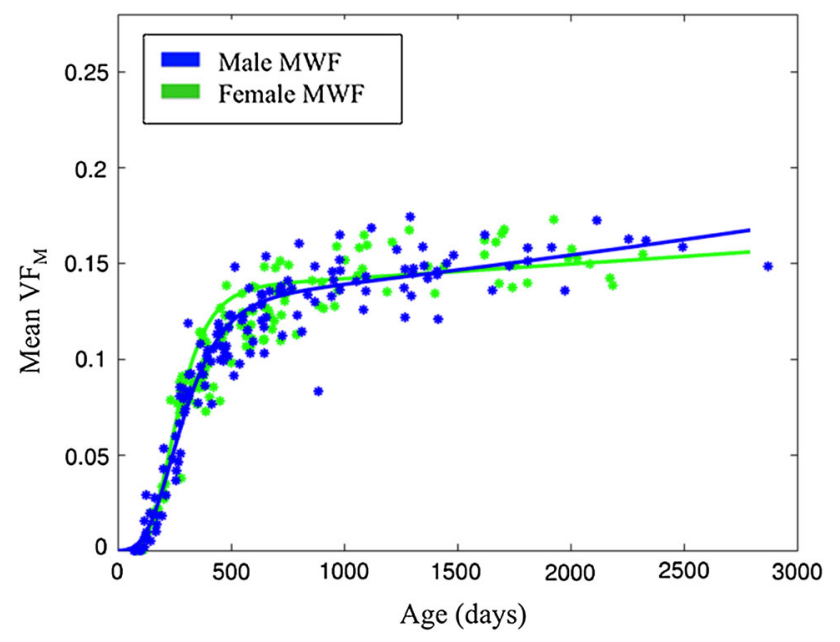

Fig. $5 \mathrm{VF}_{\mathrm{M}}$ developmental trajectory of the splenium of the corpus callosum. Trajectories are separated by gender, highlighting the developmental profile difference between males and females

Characterization of longitudinal changes of myelin content is particularly well suited to examine the spatiotemporal relationships of brain development. Myelination undergoes a dramatic increase during the first years of life in response to activity-based learning and plasticity, with the greatest rates of change occurring before 3 years of age (Yakovlev and Lecours 1967). Tracking these nonlinear changes using quantitative MRI techniques sensitive to myelination allows us to examine this neurodevelopmental process in vivo and quantitatively map the myelination trajectory (Deoni et al. 2011, 2012). Establishment of $\mathrm{VF}_{\mathrm{M}}$ growth and rate of growth trajectories offers the potential to distinguish typical and atypical myelination patterns, as well as determine the age at which such deviations from the normal trajectory occur (Dean et al. 2014a, b). This is increasingly important to the study of neurobehavioral and neuropsychiatric disorders, as many of the pathological features of these diseases are consistent with disrupted or aberrant myelination (Fields 2008).

Modeling of the nonlinear white matter maturation provides a more detailed examination of the microstructural changes that take place during neurodevelopment. Such analysis provides a unique parameterization of the developmental trajectory, yielding intuitive growth parameters related to the underlying development (Sadeghi et al. 2013). Each of these parameters has a distinctive role on the overall curve (Supplementary Fig. S3), and therefore, interrogation of these parameters may be useful in future investigations. Specifically, the modified Gompertz growth function (Eq. 1) applied throughout this work enables the trajectory to be put in terms of amplitude $(\alpha)$, lag $(\beta)$, and growth rates $(\gamma, \delta)$. While the use of a three-parameter Gompertz function $\left(f(t)=\alpha \exp \left(-\beta \exp \left(-\gamma_{t}\right)\right)\right)$ has previously been used to
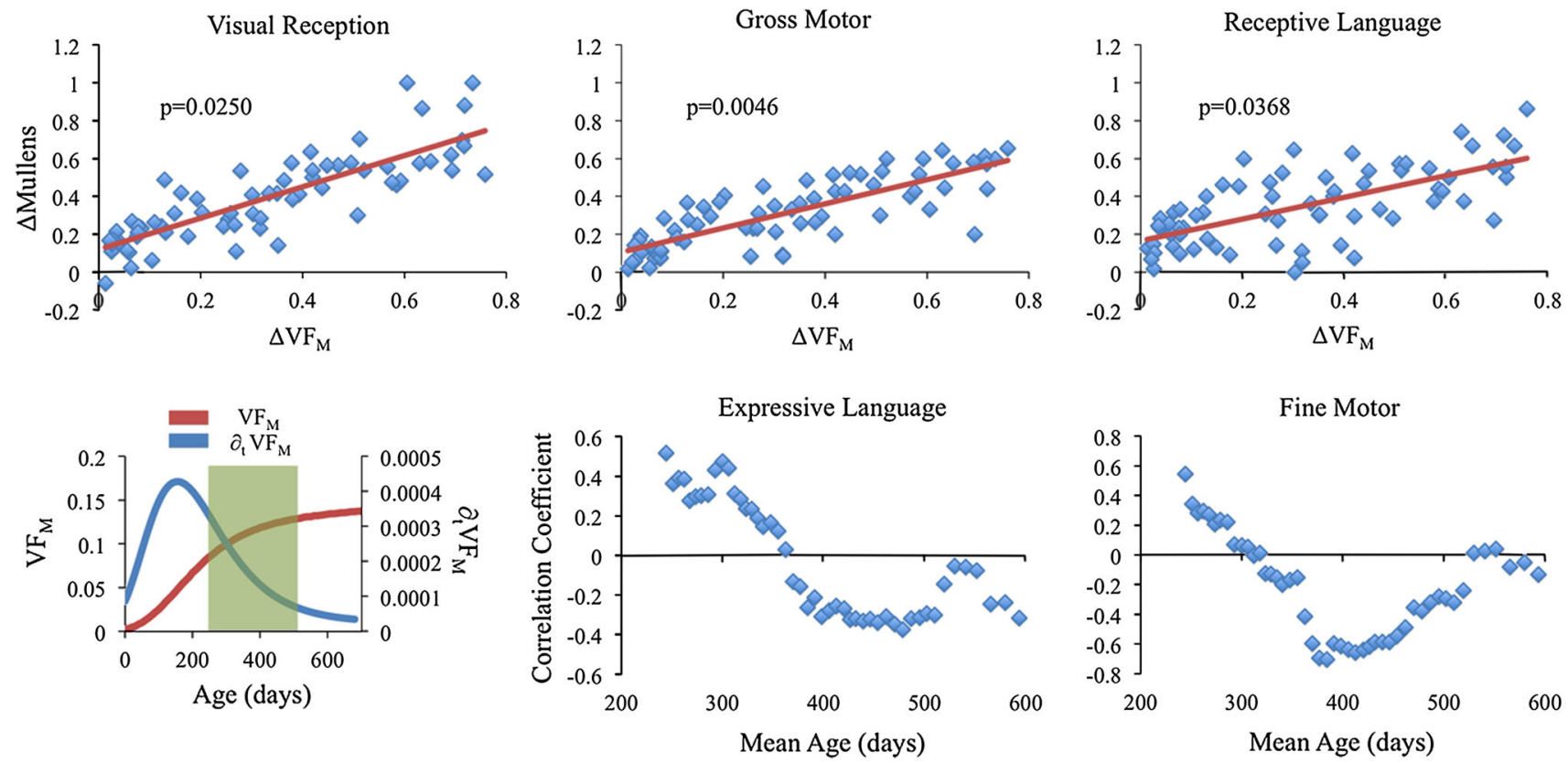

Fig. 6 Top row: change in visual reception, gross motor, and receptive language Mullen scales of early learning assessment scores as a function of changing $\mathrm{VF}_{\mathrm{M}}$ for the thalamus. The change in cognitive assessment scores were found to significantly correlate with changing $\mathrm{VF}_{\mathrm{M}}$, suggesting concomitant structure-function development. Bottom row: illustrative moving average correlations (Pearson's r, $y$-axis) as a function of mean age (days, $x$-axis) for the thalamus. Plots demonstrate the dynamic relationships between $\Delta \mathrm{VF}_{\mathrm{M}}$ and fine motor and expressive language $\Delta_{\mathrm{M}}$. The age range at which these relationships transition appears to overlap with changes in the $\mathrm{VF}_{\mathrm{M}}$ and $\partial_{\mathrm{t}} \mathrm{VF}_{\mathrm{M}}$ trajectories 
characterize the development of DT-MRI measures, including FA, MD, and RD, in young children (Sadeghi et al. 2013) the function described in these works are limited to describing continued developmental changes observed to take place in adolescence and adulthood (Lebel and Beaulieu 2011) due to the asymptotic nature of the function. Due to the additional model parameter, the modified Gompertz function allows for continued growth and has been shown to outperform other growth models, including the threeparameter Gompertz function, at characterizing observed patterns of cross-sectional $\mathrm{VF}_{\mathrm{M}}$ development (Dean et al. 2014a). Nonetheless, while these two mathematically similar models reflect the development of different MRI metrics, comparison of the Gompertz parameters from these separate but complementary models may be interesting to examine the relationships between DT-MRI and MCR metrics in neurodevelopment.

In addition to comparing growth models constructed from differing imaging techniques, a more complete characterization of underlying microstructural white matter and myelin development may be realized by integrating these distinct quantitative imaging methods into a single neuroimaging protocol. Development of such a protocol would require optimization of the scan pulse sequences for children (Deoni et al. 2012) and scan acquisition times would need to be considered, as longer scans can make acquiring artifact-free MR images from children more challenging (Dean et al. 2014b). Though we are unaware of any current study that incorporates this multimodal information, combining MCR with DT-MRI, MT-imaging measures, as well as metrics of cortical thickness (CT) and functional brain activity (fMRI and/or EEG) would be informative to the study of brain development. Such a study would allow the relationships between these separate measures to be investigated as well as provide a more thorough description of the structural and functional changes that take place as the brain matures.

In addition, mixed effects modeling of longitudinal data is advantageous to other cross-sectional modeling techniques at characterizing brain development due to its ability to provide information that is relevant to population and individual growth. Mixed effects methods provide a more flexible modeling framework, as repeated measurements from the same individual are easily handled and missing observations from an individual do not require the entire set of individual measurements to be removed from the analysis. The success of these types of mixed model designs has been demonstrated in studies examining brain development in young children (Sadeghi et al. 2013) and adolescents (Lebel and Beaulieu 2011). Our results complement these previous studies to provide the first mixed effects analysis of $\mathrm{VF}_{\mathrm{M}}$ development, allowing for the creation of population and individual $\mathrm{VF}_{\mathrm{M}}$ developmental trajectories for the first time. Establishment of such individual $\mathrm{VF}_{\mathrm{M}}$ growth trajectories may provide the ability to pinpoint subtle individual variations of white matter development. This feature of mixed effects models is essential for their continued use in neuroimaging and identifying specific characteristics within individuals who go on to develop neurodevelopmental disorders (Xu et al. 2008).

Developmental trajectories are often reported to differ between sexes. Lenroot et al. (2007) reported distinguishable longitudinal patterns of brain development between males and females, observing males to have accelerated white matter development when compared to females. Surprisingly, comparison of the overall trajectories between males and females yielded no significant differences in any of the examined regions. Such findings may be a result of the region of interest-type of analysis performed, in which subtle trajectory differences between males and females were masked by averaging large numbers of voxels. Extension of the regional analysis to a voxelwise inspection may be more informative and unmask subtle sex differences. Nevertheless, pairwise comparison of the modified Gompertz modeling parameters revealed significant differences in the majority of regions (Supplementary Table 4). These differences may be interpreted in terms of the defining characteristics that each parameter represents in the modified Gompertz model. For example, the parameters $\gamma$ and $\delta$ represent $\mathrm{VF}_{\mathrm{M}}$ growth rates in the modified Gompertz model and, therefore, our analysis suggests that the majority of $\mathrm{VF}_{\mathrm{M}}$ growth rates were significantly higher in males than females. Females were found to have significantly larger $\alpha$ values in most of the examined regions, suggesting the amplitude of the developmental trajectory to be larger in females. These results are consistent with previous studies that have reported males to have steeper rates of increase of white matter volume (Lenroot et al. 2007) while females have been observed to have higher FA values relative to males in adolescents (Bava et al. 2008). While the cause for these sex differences is unknown, it has been hypothesized elsewhere that varying exposure levels to sex hormones during development may influence the maturational trajectory of the brain (Neufang et al. 2009). Other extraneous factors, including socioeconomics and genetic makeup, are also likely to affect the developmental trajectory of the brain (Giedd and Rapoport 2010) and thus further examination of the role of these characteristics on sexual dimorphic neurodevelopment is essential.

Myelination of white matter pathways is an integral component to the development of cognitive function during childhood, as it allows for faster electrical conduction and 
therefore more efficient communication in the brain. Based on the results from previous neuroimaging studies (Dubois et al. 2008; Short et al. 2013), we anticipated on finding relationships between longitudinal measures of $\mathrm{VF}_{\mathrm{M}}$ and Mullen assessment measures. Our results show longitudinal changes of $\mathrm{VF}_{\mathrm{M}}$ to positively correlate with changes in gross motor, receptive language, and visual reception, while the correlation between $\mathrm{VF}_{\mathrm{M}}$ and scales of expressive language and fine motor was found to change with age. These results are suggestive that individual differences in the rate of $\mathrm{VF}_{\mathrm{M}}$ change over time are associated with individual differences in the rate of behavioral change over time. Moreover, the relationships between these structural and functional changes are dynamically influenced by age (as illustrated in Fig. 6). Such a result alludes to the time interval between 1 and 2 years of age as representing a transitionary period of brain maturation, a particularly important stage of development that has been previously been indicated as a time in which neurodevelopmental disorders, such as autism, manifest (Courchesne et al. 2007; Bale et al. 2010). These results are additionally consistent with the interpretations of previous works in that there exists an interdependent relationship between cognitive and behavioral functions and the myelination of interconnected brain networks that depend on the rapid transmission of information (Casey et al. 2005; Fields 2008; Tamnes et al. 2010). However, while this interpretation seems plausible given our observations, studies investigating the extrinsic and intrinsic factors that influence myelination are needed to further examine this hypothesis.

Of the five Mullen scales, changes in gross motor ability correlated with changes in $\mathrm{VF}_{\mathrm{M}}$ in the most number of regions (22 of 28). Developing gross motor ability is central to the development of skills and abilities that are related to large movements (i.e., crawling, walking, head control, posture) and also play a primary role in the other four cognitive domains (Mullen 1995). These abilities require a foundation of evolved communication pathways and may be the reason why this measure is observed to be correlated with the numerous brain regions. Other positive correlations found between the changes in visual reception and $\mathrm{VF}_{\mathrm{M}}$ involved regions (thalamus, internal capsule, corona radiata) associated with the central visual pathways, signifying the myelination of these areas lead to improved visual processing skills. Likewise, regions correlated with changes in receptive language, including cerebellar white matter, thalamus, posterior internal capsule, superior longitudinal fasciculus, have previously been identified to be associated with changes in language comprehension (Geschwind 1970; Bernal and Altman 2010). Although these results are preliminary, our data lend itself to further investigation of these relationships. In particular, examining the ability of maturing white matter to predict subsequent functional connectivity could prove to be informative in understanding how variations of the structural properties of white matter systematically influence cognitive function. Moreover, as the integration of functional resting state data and/or EEG data with quantitative MRI measurements has recently been successful at examining the functional maturation of white matter (Dubois et al. 2008), incorporating these additional functionally relevant data with $\mathrm{VF}_{\mathrm{M}}$ measurements would likely be beneficial to understanding the structure-function relationships of the developing brain.

Though mcDESPOT is a recent MCR imaging technique that affords rapid, whole-brain coverage, the imaging technique differs from conventional and established multispin echo methods (Zhang et al. 2014). In particular, conventional $\mathrm{VF}_{\mathrm{M}}$ derived from multi-echo spin echo measurements has shown to strongly correlate to histological measurements of myelin content (Laule et al. 2008) and provide a more specific marker for myelin than parameters derived from diffusion tensor (Mädler et al. 2008) and magnetization transfer imaging (Vavasour et al. 1998). Histological comparisons of mcDESPOT $\mathrm{VF}_{\mathrm{M}}$ measures have shown strong qualitative agreement in the Shaking Pup model of dysmyelination (Hurley et al. 2010), though quantitative comparisons remain to be carried out. Prior mcDESPOT studies of neurodevelopment have reproduced the known histologically established spatialtemporal pattern of myelination, and $\mathrm{VF}_{\mathrm{M}}$ measures have been shown to reflect clinical disability in known demyelinating disorders, including multiple sclerosis (Kitzler et al. 2012; Kolind et al. 2012) and amyotrophic lateral sclerosis (Kolind et al. 2013). Such comparisons give confidence that if mcDESPOT $\mathrm{VF}_{\mathrm{M}}$ measures are not uniquely specific to myelin, they provide unique information that is strongly sensitive to myelin content. Still, additional studies examining the specificity of mcDESPOT-derived $\mathrm{VF}_{\mathrm{M}}$ measures to myelin, including quantitative comparison to histological stains of myelin content, are necessary to validate the mcDESPOT technique as a viable "myelin imaging" technique. These studies are of great interest and will be beneficial as an area of future research.

The region of interest-based analyses performed in this work are useful for characterizing the average developmental trajectory of specific, anatomically defined brain regions; however, voxel-based methods may be more revealing as these methods provide information across the whole brain. Performing the mixed effects modeling of our longitudinal data at the voxel level would provide estimates of the overall population (fixed effects) and subject-specific (random effects) modified Gompertz parameters at each imaging voxel, allowing us to predict $\mathrm{VF}_{\mathrm{M}}$ values at later ages across the whole brain. This is important for 
establishing a normative model of neurodevelopment and having the ability to identify deviations from typical growth as these differences are unlikely to be restricted to grossly defined regions of interest (Snook et al. 2007). For this reason, future work extending the nonlinear mixed effects modeling to cover the whole brain is planned.

In summary, we have presented the first longitudinal examination of white matter development throughout early childhood. The results reported from previous cross-sectional studies of $\mathrm{VF}_{\mathrm{M}}$ maturation are extended by showing longitudinally that $\mathrm{VF}_{\mathrm{M}}$ follows a specific, nonlinear trajectory throughout early childhood and is qualitatively consistent with spatial-temporal patterns of myelination of the human brain. We have shown that $\mathrm{VF}_{\mathrm{M}}$ trajectories of the normative population and individual subjects can be characterized and predicted by modeling for the nonlinear growth patterns using a nonlinear mixed effects framework. Moreover, these $\mathrm{VF}_{\mathrm{M}}$ developmental trajectories differed between males and females, while changes in $\mathrm{VF}_{\mathrm{M}}$ were significantly correlated with changing measures of cognition and behavior. The coupling between this structural and functional development provides insight into the evolving relationship between myelin content and behavior as well as indicating evidence of sensitive periods of neurodevelopment between 1 and 2 years of age. This presented work provides an important step for understanding the typical patterns of normative white matter maturation and its relationship to emerging cognition as well as providing a foundation for future studies examining aberrant brain development.

Acknowledgments We thank all of the families who donated their time for taking part in this research. This work was supported by the National Institutes of Mental Health (R01 MH087510). JOM is supported by a Sir Henry Wellcome Postdoctoral Fellowship awarded by the Wellcome Trust (No 096195).

Open Access This article is distributed under the terms of the Creative Commons Attribution License which permits any use, distribution, and reproduction in any medium, provided the original author(s) and the source are credited.

\section{References}

Aubert-Broche B, Fonov VS, García-Lorenzo D, Mouiha A, Guizard N, Coupé P, Eskildsen SF, Collins DL (2013) A new method for structural volume analysis of longitudinal brain MRI data and its application in studying the growth trajectories of anatomical brain structures in childhood. Neuroimage 82:393-402

Avants BB, Epstein CL, Grossman M, Gee JC (2008) Symmetric diffeomorphic image registration with cross-correlation: evaluating automated labeling of elderly and neurodegenerative brain. Med Image Anal 12:26-41

Bale TL, Baram TZ, Brown AS, Goldstein JM, Insel TR, McCarthy MM, Nemeroff CB, Reyes TM, Simerly RB, Susser ES, Nestler
EJ (2010) Early life programming and neurodevelopmental disorders. Biol Psychiatry 68:314-319

Bava S, Boucquey V, Goldenberg D, Thayer RE, Ward M, Jacobus J, Tapert SF (2008) Sex differences in adolescent white matter architecture. Brain Res 1375:41-48

Bernal B, Altman N (2010) The connectivity of the superior longitudinal fasciculus: a tractography DTI study. Magn Reson Imag 28:217-225

Casey B, Tottenham N, Liston C, Durston S (2005) Imaging the developing brain: what have we learned about cognitive development? Trends Cogn. Sci (Regul Ed) 9:104-110

Courchesne E, Pierce K, Schumann C, Redcay E (2007) Mapping early brain development in autism. Neuron 56:399-413

Dean DC III, O'Muircheartaigh J, Dirks H, Waskiewicz N, Lehman K, Walker L, Han M, Deoni SC (2014a) Modeling healthy male white matter and myelin development: 3 through 60 months of age. Neuroimage 84:742-752

Dean DC III, Dirks H, O'Muircheartaigh J, Walker L, Jerskey BA, Lehman K, Han M, Waskiewicz N, Deoni SC (2014b) Pediatric neuroimaging using magnetic resonance imaging during nonsedated sleep. Pediatr Radiol 44:64-72

Deoni SC (2010) Correction of main and transmit magnetic field (B0 and B1) inhomogeneity effects in multicomponent-driven equilibrium single-pulse observation of T1 and T2. Magn Reson Med 65:1021-1035

Deoni SC, Rutt BK, Arun T, Pierpaoli C, Jones DK (2008) Gleaning multicomponent $\mathrm{T} 1$ and $\mathrm{T} 2$ information from steady-state imaging data. Magn Reson Med 60:1372-1387

Deoni SC, Mercure E, Blasi A, Gasston D, Thomson A, Johnson M, Williams SC, Murphy DG (2011) Mapping infant brain myelination with magnetic resonance imaging. J Neurosci 31:784-791

Deoni SC, Dean DC III, O'Muircheartaigh J, Dirks H, Jerskey BA (2012) Investigating white matter development in infancy and early childhood using myelin water faction and relaxation time mapping. Neuroimage 63:1038-1053

Deoni SC, Matthews L, Kolind SH (2013) One component? Two components? Three? The effect of including a nonexchanging 'free' water component in multicomponent driven equilibrium single pulse observation of T1 and T2. Magn Reson Med 70:147-154

Dubois J, Dehaene-Lambertz G, Soarès C, Cointepas Y, Le Bihan D, Hertz-Pannier L (2008) Microstructural correlates of infant functional development: example of the visual pathways. J Neurosci 28:1943-1948

Durston S, Casey B (2006) What have we learned about cognitive development from neuroimaging? Neuropsychologia 44:2149-2157

Fields RD (2008) White matter in learning, cognition and psychiatric disorders. Trends Neurosci 31:361-370

Flechsig P (1901) Developmental (myelogenetic) localisation of the cerebral cortex in the human subject. Lancet 158:1027-1030

Geschwind (1970) The organization of language and the brain. Science 170:940-944

Giedd JN, Rapoport JL (2010) Structural MRI of pediatric brain development: what have we learned and where are we going? Neuron 67:728-734

Giedd J, Snell J, Lange N, Rajapakse J, Casey B, Kozuch P, Vaituzis A, Vauss Y, Hamburger S, Kaysen D, Rapoport J (1996) Quantitative magnetic resonance imaging of human brain development: ages 4-18. Cereb Cortex 6:551-560

Giedd J, Blumenthal J, Jeffries N (1999) Brain development during childhood and adolescence: a longitudinal MRI study. Nature 2:861-863

Gilmore JH, Shi F, Woolson SL, Knickmeyer RC, Short SJ, Lin W, Zhu H, Hamer RM, Styner M, Shen D (2012) Longitudinal development of cortical and subcortical gray matter from birth to 2 years. Cereb Cortex 22:2478-2485 
Holm S (1979) A simple sequentially rejective multiple test procedure. Scand J Stat 6:65-70

Hüppi PS (2008) Neuroimaging of brain development-discovering the origins of neuropsychiatric disorders? Pediatr Res 64:325

Hurley SA, Mossahebi P, Samsonov AA, Alexander AL, Deoni SC, Fisher R, Duncan ID, Field AS (2010) Multicomponent relaxometry (mcDESPOT) in the shaking pup model of dysmyelination. In: Proceeding of the 18th Annual Meeting of the ISMRM, 4516

Jones DK, Knösche T, Turner R (2013) White matter integrity, fiber count, and other fallacies: the do's and don'ts of diffusion MRI. Neuroimage 73:239-254

Kitzler HH, Su J, Zeineh M, Harper-Little C, Leung A, Kremenchutzky M, Deoni SC, Rutt BK (2012) Deficient MWF mapping in multiple sclerosis using 3D whole-brain multicomponent relaxation MRI. Neuroimage 59:2670-2677

Kolind S, Matthews L, Johansen-Berg H, Leite MI, Williams SC, Deoni S, Palace J (2012) Myelin water imaging reflects clinical variability in multiple sclerosis. Neuroimage 60:263-270

Kolind S, Sharma R, Knight S, Johansen-Berg H, Talbot K, Turner MR (2013) Myelin imaging in amyotrophic and primary lateral sclerosis. Amyotroph Lateral Scler Frontotemporal Degener $14: 562-573$

Laule C et al (2008) Myelin water imaging of multiple sclerosis at 7 T: correlations with histopathology. Neuroimage 40:1575-1580

Lebel C, Beaulieu C (2011) Longitudinal development of human brain wiring continues from childhood into adulthood. J Neurosci 31:10937-10947

Lebel C, Walker L, Leemans A, Phillips L, Beaulieu C (2008) Microstructural maturation of the human brain from childhood to adulthood. Neuroimage 40:1044-1055

Lenroot RK, Gogtay N, Greenstein DK, Wells EM, Wallace GL, Clasen LS, Blumenthal JD, Lerch J, Zijdenbos AP, Evans AC, Thompson PM, Giedd JN (2007) Sexual dimorphism of brain developmental trajectories during childhood and adolescence. Neuroimage 36:1065-1073

Lindstrom MJ, Bates DM (1990) Nonlinear mixed effects models for repeated measures data. Biometrics 46:673-687

MacKay A, Whittall K, Adler J, Li D, Paty D, Graeb D (1994) In vivo visualization of myelin water in brain by magnetic resonance. Magn Reson Med 31:673-677

Mädler B, Drabycz S, Kolind S, Whittall K (2008) Is diffusion anisotropy an accurate monitor of myelination?: correlation of multicomponent $\mathrm{T} 2$ relaxation and diffusion tensor anisotropy in human brain. Magn Reson Imaging 26:874-888

Mazziotta J, Toga A, Evans A, Fox P, Lancaster J, Zilles K, Woods R, Paus T, Simpson G, Pike B, Holmes C, Collins L, Thompson P, MacDonald D, Iacoboni M, Schormann T, Amunts K, PalomeroGallagher N, Geyer S, Parsons L, Narr K, Kabani N, Le Goualher G, Boomsma D, Cannon T, Kawashima R, Mazoyer B (2001) A probabilistic atlas and reference system for the human brain: international Consortium for Brain Mapping (ICBM). Philos Trans R Soc Lond B Biol Sci 356:1293-1322

Mori S, Oishi K, Faria AV (2009) White matter atlases based on diffusion tensor imaging. Curr Opin Neurol 22:362-369

Mullen E (1995) Mullen scales of early learning, AGS edn. American Guidance Service Inc, Circle Pines MN
Neufang S, Specht K, Hausmann M, Güntürkün O, HerpertzDahlmann B, Fink GR, Konrad K (2009) Sex differences and the impact of steroid hormones on the developing human brain. Cereb Cortex 19:464-473

O'Muircheartaigh J, Dean DC 3rd, Dirks H, Waskiewicz N, Lehman K, Jerskey BA, Deoni SC (2013) Interactions between white matter asymmetry and language during neurodevelopment. J Neurosci 33:16170-16177

Paus T, Collins DL, Evans AC, Leonard G, Pike B, Zijdenbos A (2001) Maturation of white matter in the human brain: a review of magnetic resonance studies. Brain Res Bull 54:255-266

Pujol J, Soriano-Mas C, Ortiz H, Sebastián-Gallés N, Losilla JM, Deus J (2006) Myelination of language-related areas in the developing brain. Neurology 66:339-343

Rademacher J, Engelbrecht V, Bürgel U, Freund HJ, Zilles K (1999) Measuring in vivo myelination of human white matter fiber tracts with magnetization transfer MR. Neuroimage 9:393-406

Sadeghi N, Prastawa M, Fletcher PT, Wolff J, Gilmore JH, Gerig G (2013) Regional characterization of longitudinal DT-MRI to study white matter maturation of the early developing brain. Neuroimage 68:236-247

Short SJ, Elison JT, Goldman BD, Styner M, Gu H, Connelly M, Maltbie E, Woolson S, Lin W, Gerig G, Reznick JS, Gilmore JH (2013) Associations between white matter microstructure and infants' working memory. Neuroimage 64:156-166

Smith SM, Jenkinson M, Woolrich MW, Beckmann CF, Behrens TE, Johansen-Berg H, Bannister PR, De Luca M, Drobnjak I, Flitney DE, Niazy RK, Saunders J, Vickers J, Zhang Y, De Stefano N, Brady JM, Matthews PM (2004) Advances in functional and structural MR image analysis and implementation as FSL. Neuroimage 23(Suppl 1):S208-S219

Snook L, Plewes C, Beaulieu C (2007) Voxel based versus region of interest analysis in diffusion tensor imaging of neurodevelopment. Neuroimage 34:243-252

Tamnes CK, Ostby Y, Walhovd KB, Westlye LT, Due-Tonnessen P, Fjell AM (2010) Intellectual abilities and white matter microstructure in development: a diffusion tensor imaging study. Hum Brain Mapp 31:1609-1625

Vavasour I, Whittall K, Mackay A (1998) A comparison between magnetization transfer ratios and myelin water percentages in normals and multiple sclerosis patients. Magn Reson Med 40:763-768

Xu S, Styner M, Gilmore J, Piven J, Gerig G (2008) Multivariate nonlinear mixed model to analyze longitudinal image data: MRI study of early brain development, In: MMBIA. IEEE Computer Society, pp 1-8

Yakovlev PI, Lecours AR (1967) The myelogenetic cycles of regional maturation of the brain. In: Mankowski A (ed) Regional development of the brain in early life. David, Philadelphia, pp 3-69

Zhang J, Kolind SH, Laule C, MacKay AL (2014) Comparison of myelin water fraction from multiecho $\mathrm{T}_{2}$ decay curve and steady-state methods. Magn Reson Med (in press) 